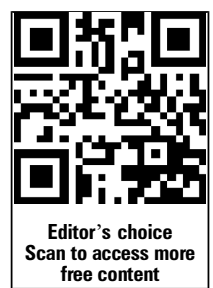

Division of Cardiovasculas

Medicine, Mayo Clinic, Rochester, Minnesota, USA ${ }^{2}$ Department of Cardiology, Policlinico Hospital, Modena and Reggio Emilia University, Modena, Italy

${ }^{3}$ Department of Cardiac,

Thoracic and Vascular Sciences, School of Medicine, University

of Padua, Padua, Italy

\section{Correspondence to}

Dr Maurice Enriquez-Sarano,

Division of Cardiovascular

Diseases, Mayo Clinic, 200

First Street SW, Rochester, MN

55905, USA:

sarano.maurice@mayo.edu

Received 30 October 2014 Revised 17 March 2015 Accepted 27 March 2015 Published Online First

2 May 2015

\section{SLinked}

- http://dx.doi.org/10.1136/ heartjnl-2015-307759

\section{CrossMark}

\author{
To cite: Mantovani $F$, \\ Clavel M-A, Vatury O, et al. \\ Heart 2015;101:
}

1111-1117

\title{
Cleft-like indentations in myxomatous mitral valves by three-dimensional echocardiographic imaging
}

\author{
Francesca Mantovani, ${ }^{1,2}$ Marie-Annick Clavel, ${ }^{1}$ Ori Vatury, ${ }^{1}$ Rakesh M Suri, ${ }^{1}$ \\ Sunil V Mankad, ${ }^{1}$ Joseph Malouf, ${ }^{1}$ Hector I Michelena, ${ }^{1}$ Sonia Jain, ${ }^{1}$ \\ Luigi Paolo Badano, ${ }^{3}$ Maurice Enriquez-Sarano ${ }^{1}$
}

\begin{abstract}
Objectives Cleft-like indentations (CLI) are deep separations between scallops of the mitral posterior leaflet observed in myxomatous mitral valve disease (MMVD), but their diagnosis, mechanisms and implications are unknown. Using 3D transoesophageal echocardiography (3DTOC), we aimed at assessing diagnostic accuracy and defining mechanisms of CLI in patients undergoing surgery for MMVD.
\end{abstract}

Methods 3DTOC of mitral valve was acquired in 49 patients with MMVD and severe regurgitation prior to valve repair. Qualitative review compared 3DTOC diagnosis of CLI with surgical inspection. Mitral, annular and leaflet dimensions were quantified with dedicated software and compared between those with and without CLI.

Results Diagnosis of CLI was made by 3DTOC in $17(35 \%)$ while none was identified by $2 \mathrm{D}$ and was confirmed in $15(88 \%)$ by surgical inspection. Mechanistically, LV diameters and mitral regurgitant volume (RVol) were similar with and without CLI $(p>0.49)$. Conversely, mitral annulus was smaller with CLI (anteroposterior diameter $42.2 \pm 7.1$ vs 47.0 $\pm 7.5 \mathrm{~mm}, \mathrm{p}=0.04$; circumference $133 \pm 16$ vs 148 $\pm 19 \mathrm{~mm}, p=0.009$; area $1289 \pm 326$ vs $1619 \pm 427 \mathrm{~mm}^{2}$, $\mathrm{p}=0.008)$. Prolapse volume tended to be smaller with CLI (1.9 \pm 1.2 vs $4.0 \pm 4.3 \mathrm{~mL}, \mathrm{p}=0.06)$ involving single posterior scallop at surgery ( $82 \%$ vs $44 \%, p=0.007)$ with smaller 3DTOC leaflet area (1574 \pm 409 vs $\left.2019 \pm 652 \mathrm{~mm}^{2}, p=0.01\right)$. During valve repair, surgical closure of all surgically diagnosed CLI was required.

Conclusions Posterior leaflet $\mathrm{CLI}$ are frequent in MMVD, are identified by 3DTOC with high accuracy and require closure during valve repair. CLI are mechanistically not related to excess annular enlargement or excess prolapse. Conversely, CLI occur in the context of single scallop prolapse with tissue paucity causing excess separation of scallops. These 3DTOC data enhance diagnostic and mechanistic comprehension of the diversity of MMVD phenotypical presentation.

\section{INTRODUCTION}

Myxomatous mitral valve disease (MMVD) is the leading cause of organic severe mitral regurgitation (MR) in western countries and frequently requires surgical treatment. ${ }^{1}$ Mitral valve repair is the procedure of choice for patients with MMVD because it can restore normal life expectancy when timely performed. ${ }^{2}$ However, the success of mitral valve repair, defined by the ability to perform the procedure during surgery and by long-term durability, ${ }^{3}$ depends on the congruence of surgeon's expertise with specific lesions to be repaired. ${ }^{4}$ Predicting successful repair is crucial to indicating surgery in most recent guidelines ${ }^{5}$ and demands accurate mitral anatomic assessment and characterisation of leaflets' lesions. ${ }^{6}$

3D transoesophageal echocardiography (3DTOC) profoundly changed the ability to visualise the complete mitral valve anatomy in vivo ${ }^{7}$ while gaps in mitral tissue are better diagnosed. True posterior leaflet clefts, cleaving it in two equal remnants, while long-known, ${ }^{9}$ are rare, but have recently been diagnosed in vivo with 3DTOC. ${ }^{10} 11$ Lately, another feature of MMVD was described: deep 'clefts' of posterior mitral leaflet, ${ }^{12}$ which do not really cleave the leaflet, but occupy the position of normal indentations between its three scallops. Normal indentations are usually not deep, but abnormal indentations, although different from true clefts, ${ }^{9}$ form when they are deep cleft-like indentations (CLI) that can be associated with $\mathrm{MR}^{13}$ and failure of mitral repair. ${ }^{14}$

These recently recognised CLI have been hypothesised as linked to mitral prolapse extent ${ }^{12}$ and not to MR per se. Whether annular enlargement, tissue excess or prolapse severity are associated with CLI presence is unknown, and little is known about CLI mechanism. Therefore, we initiated a prospective 3DTOC study of patients with MMVD undergoing mitral repair for MR, aiming at defining accuracy of CLI preoperative diagnosis and at defining 3D MMVD morphological features independently linked to CLI presence. Our working mechanistic hypothesis was that excess annular enlargement, by separating posterior mitral scallops, was CLI's main causal determinant.

\section{METHODS}

The study included 49 consecutive patients referred for elective surgical mitral valve repair for MMVD with high-quality 3DTOC quantifiable. Patients were enrolled prospectively if they had (1) severe MR due to MMVD, (2) indicating mitral valve repair, (3) comprehensive transthoracic Doppler echocardiography performed at Mayo Clinic during the same episode of care, (4) 2D and 3D intraoperative transoesophageal echocardiography (TEE) of mitral valve and (5) 3D datasets of sufficient quality to quantify mitral annulus and leaflets alterations. Exclusion criteria were contraindications to TEE, 
uncontrolled atrial fibrillation, mitral stenosis, moderate-tosevere aortic valve disease, moderate-to-severe tricuspid regurgitation or associated pericardial or congenital heart disease. Patients with MMVD associated with connective tissue disorders, Marfan syndrome, hypertrophic cardiomyopathy and rheumatic heart disease were also excluded. The study was approved by Mayo Institutional Review Board and was deemed low-risk, requiring only verbal consent.

\section{Doppler and 2D echocardiographic measurements}

Comprehensive Doppler and 2D echocardiography data were prospectively recorded preoperatively. LV, left atrium (LA) dimensions and LVEF were measured by 2D echocardiography. MR was quantified ${ }^{15}$ as effective regurgitant orifice (ERO) and regurgitant volume (RVol).

\section{DTOC qualitative and quantitative image analysis}

TEE was performed intraoperatively, after initial anaesthesia induction and endotracheal intubation, before cardiopulmonary bypass or thoracic incisions. The ultrasound platform was RT3DE imaging probe (model X7-2t) and iE33 echocardiography imaging platform (Philips Medical Systems, Bothell, Washington, USA).

From mid-oesophageal position, live 3DTOC zoom of mitral valve included aortic valve and entire mitral annulus throughout the cardiac cycle. For mitral qualitative assessment, comprehensive imaging was obtained by standard 2DTEE and by 3DTOC after cropping. 3DTOC was recorded both as live 3D (singlebeat) and as multi-beat full volume datasets, and imaging was optimised to enhance physical and temporal $(8-12 \mathrm{~Hz})$ resolution. For 3DTOC interpretation, both atrial and ventricular views of the mitral valve were analysed in detail, with swivel examination of the entire valve to examine all three scallops of posterior leaflet and of indented zones between scallops. Diagnosis of CLI by echocardiography was defined as a visible tissue defect seen during systole, occupying the interscallop region of the posterior leaflet-not plicatures of leaflets' free edge at other location-(figure 1) and extending at least half depth of the adjacent mitral scallop. ${ }^{12} 16$ The ventricular aspect of mitral valve was also examined for careful assessment of mitral scallops with possible visualisation of CLI from this orientation (figure 2). The diagnostic views were stored for off-line interpretation before left atrial opening by the surgeon who directly examined the mitral valve (figure 1).

For quantitative analysis of mitral valve, full-volume 3D datasets were digitally stored and transferred to a workstation with Q-Laboratory (QLAB) Mitral Valve Quantification Software (Philips Medical Systems) for off-line analysis by an observer blinded to any preoperative and intraoperative Doppler-echocardiographic and surgical findings. After storage of 3D datasets, quantitative analysis was promptly performed. Three orthogonal mitral annulus images were displayed and subsequently modified to optimise visualisation of the entire annulus. Mitral annular measurements (annular area, circumference, intercommissural and anteroposterior diameter and height) were obtained in systole just before aortic valve closure. Annular height, maximal instantaneous vertical distance between highest (anterior or posterior) and lowest (anterolateral or posteromedial) points, was used to compute the ratio of annular height to intercommissural diameter, a measure of annular saddle shape. ${ }^{17}$ After annular delineation, mitral leaflets were traced in multiple slices from medial to lateral commissure, and landmarks were placed at coaptation zone tips to define the entire surface of anterior and posterior leaflets. From valvular 3D data, mitral leaflet measurements (3D total valvular area, anterior leaflet area, posterior leaflet area) and quantification of leaflet excess movement in relation to annular plane (volume and height of prolapse) were also obtained at the same timing in systole (figure 3). Variability of measurements was determined by repeating measurements on stored $3 \mathrm{D}$ datasets at least 3 weeks after initial measurement by the same observer (intraobserver) and a different observer (interobserver) in 10 randomly selected cases.

\section{Clinical variables}

Baseline clinical data collected at preoperative echocardiogram and clinical visits included age, gender, weight, height, body mass index, body surface area, functional status and cardiac rhythm. Cardiovascular history and medications were determined by reviewing all medical records. Surgical variables were abstracted from surgical report dictated immediately after surgery completion, regarding CLI presence and extent (leaflets, segments) of mitral prolapse.

\section{Statistical analysis}

Data are shown as mean \pm SD for continuous variables and percentages for categorical variables. Patients were categorised according to 3DTOC presence/absence of posterior leaflet CLI and compared using $t$ tests and $\chi^{2}$ or Fisher exact tests as appropriate. For CLI diagnosis, the surgeon visual inspection (blinded to 3DTOC findings) was the diagnosis standard. For reproducibility, intraobserver and interobserver variability were calculated
Figure 1 Example of a patient with myxomatous mitral valve disease (MMVD) and CLI with recording of 3D imaging and operative view imaging. Left image: 3D transoesophageal echocardiographic view of the mitral valve from the left atrial position. Right image: direct mitral valve view during surgical inspection. The red arrow indicates the $\mathrm{CLI}$ of the posterior mitral valve leaflet; the yellow arrow head indicates the prolapsing scallop (middle) of the posterior leaflet. Note, the visible deep indentation between P2 and P3 by both 3D imaging and direct viewing of the mitral valve. CLI, cleft-like indentation.

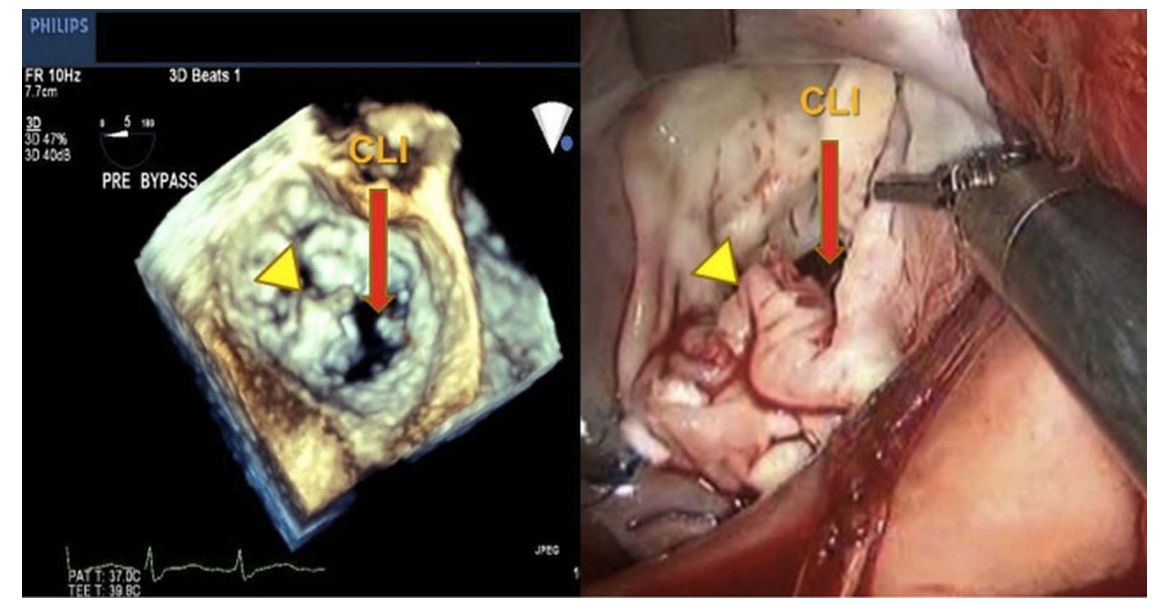


Figure 2 Example of a patient with myxomatous mitral valve disease (MMVD) and CLI with 3D imaging display from atrial and ventricular views. Mitral valve 3D

transoesophageal echocardiographic imaging viewed from left atrial (left image) and LV (right image) positions. The CLI located between P1 and P2 is indicated by a red arrow and is visible from both atrial and ventricular views. The flail segment is indicated by the yellow arrow head and the ruptured chordae by the white arrow head. Ao, aortic Valve; CLI, cleft-like indentation; LA, left atrium. LV, Left Ventricle.

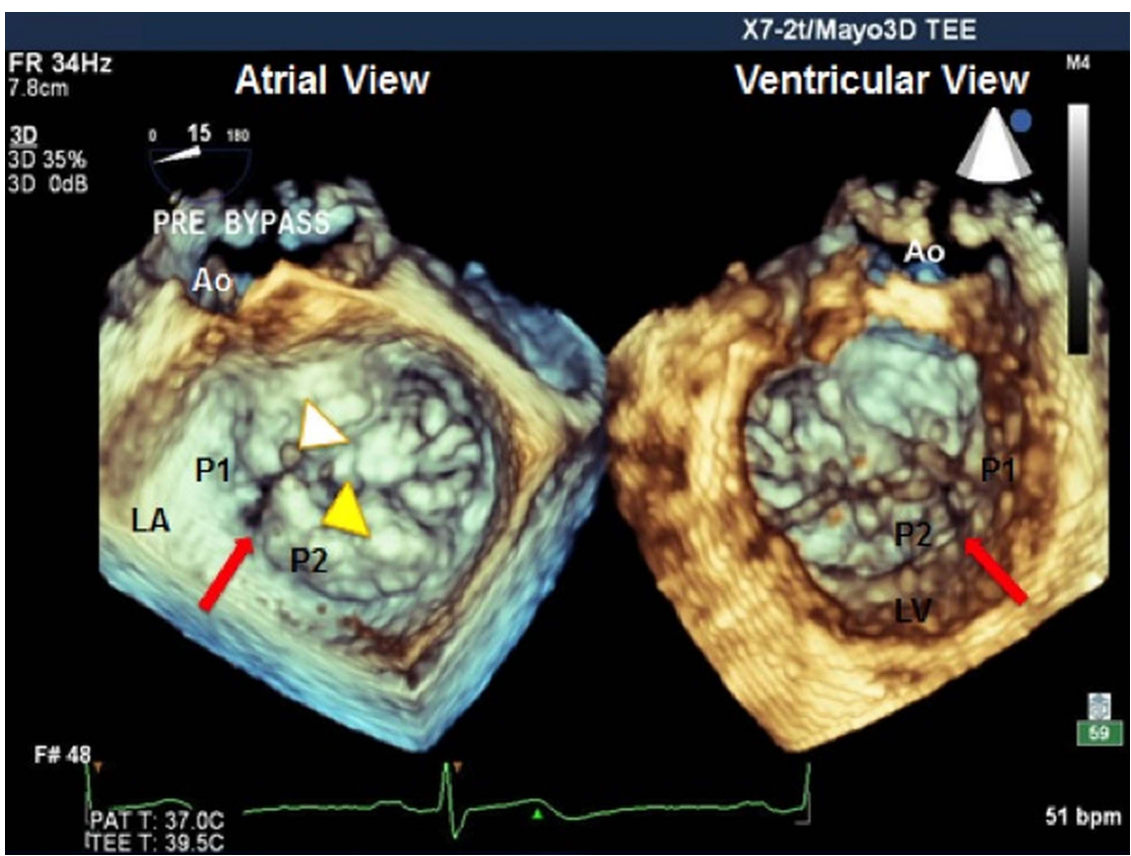

by dividing absolute differences between two measurements by their average value. All tests were two tailed; $p<0.05$ was considered significant. Analyses were performed using JMP V.9.0.1 (SAS Institute, Cary, North Carolina, USA).

\section{RESULTS}

Clinical and surgical baseline characteristics are indicated in table 1. Overall, patients presented in the typical early sixth decade with male predominance. Risk factors were typical of

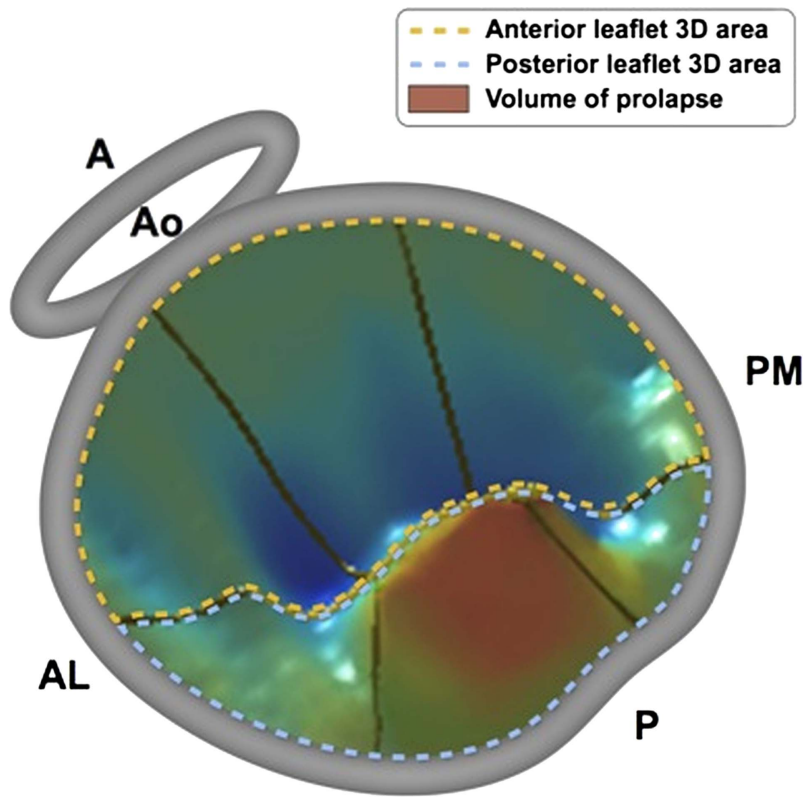

Figure $33 \mathrm{D}$ parametric models of patient's mitral valve by the quantification software (QLAB). The mitral valve is viewed from the atrial position with, in grey, the mitral and aortic (Ao) annuli. The yellow dashed line delimits the anterior leaflet, while the dashed blue line delimits the posterior leaflet of the mitral valve. Leaflet colouring in blue hues indicate that the valve is normally located on the LV side of the annulus and red hues indicate the volume of the mitral prolapsing segment. $A$, anterior; $A L$, anterolateral; $A 0$, aortic valve; $\mathrm{P}$, posterior; PM, posteromedial. age with generally normal renal function. For MR clinical manifestations, severe symptoms and atrial fibrillation were rare. Echocardiographic characteristics are presented in table 2, showing moderate LV dilatation consistent with MR associated with preserved LV function. Quantitative measurement of ERO, RVol and left atrial volume index clearly demonstrated that MR due to MMVD was severe. Bileaflet prolapse was noted in a minority of patients.

Among our 49 patients, none was diagnosed with CLI by $2 \mathrm{D}$ echo and 17 (35\%) had CLI of posterior leaflet by 3DTOC. Of these, 15 were confirmed by the surgeon, and none was surgically diagnosed in patients without 3DTOC-determined CLI. Thus, for CLI diagnosis by 3DTOC, sensitivity was $100 \%$ and specificity $94 \%$, positive predictive value $88 \%$ and negative predictive value $100 \%$. 3DTOC location of CLI was unique in 15 patients $(88 \%)$; in $8(47 \%)$, CLI were located between P1-P2 scallops, and in $7(41 \%)$ patients, CLI were located between P2-P3 scallops. Two patients had CLI of both interscallop indentations (between P1-P2 and P2-P3).

Stratified by 3DTOC-diagnosed CLI, there was no clinical difference between patients with and without CLI (table 1, right part), in particular with similar treatment in both groups proportionate to hypertension and symptoms. Among surgical variables, presence of flail segments was similar with or without CLI, but patients with CLI had more often single scallop posterior leaflet prolapse ( $82 \%$ vs $44 \%, \mathrm{p}=0.007)$.

In regard to $2 \mathrm{D}$ Doppler echocardiography, comparison between those with and without CLI yielded limited differences (table 2, right part). Dimensions of LF, LA and severity of MR were similar with or without CLI. However, patients with CLI presented with slightly lower EF $(60 \% \pm 11 \%$ vs $65 \% \pm 5 \%$, $\mathrm{p}=0.03$ ), although on average in the normal range. In all cases except one, mitral valve repair was successful (98\%) and all patients with CLI diagnosed by the surgeon underwent closure of interscallop area during repair.

The 3DTOC quantified annular and valvular variables are presented in table 3. Overall, all dimensions of annulus in systole were very similar to previous studies of MMVD undergoing surgery for MR, which had demonstrated enlargement of all dimensions in comparison to normal subjects, with mild annular 
Table 1 Clinical baseline characteristics and surgical data

\begin{tabular}{|c|c|c|c|c|}
\hline & $\begin{array}{l}\text { All patients, } \\
\mathrm{n}=49\end{array}$ & $\begin{array}{l}\text { CLI absent, } \\
n=32\end{array}$ & $\begin{array}{l}\text { CLI present, } \\
n=17\end{array}$ & p Value \\
\hline Age, years & $63 \pm 11$ & $64 \pm 10$ & $60 \pm 14$ & 0.25 \\
\hline Male gender, \% & 78 & 78 & 76 & 0.89 \\
\hline Body surface area, $\mathrm{m}^{2}$ & $1.97 \pm 0.23$ & $1.98 \pm 0.22$ & $1.95 \pm 0.26$ & 0.64 \\
\hline Body mass index, $\mathrm{kg} / \mathrm{m}^{2}$ & $26 \pm 4$ & $27 \pm 4$ & $26 \pm 3$ & 0.38 \\
\hline Dyslipidaemia, \% & 41 & 38 & 47 & 0.52 \\
\hline Hypertension, \% & 43 & 44 & 41 & 0.86 \\
\hline Diabetes, \% & 8 & 9 & 6 & 0.66 \\
\hline History of CAD, \% & 8 & 6 & 12 & 0.51 \\
\hline Atrial fibrillation, \% & 14 & 13 & 18 & 0.62 \\
\hline \multicolumn{5}{|l|}{ NYHA class, \% } \\
\hline I & 31 & 34 & 24 & 0.86 \\
\hline II & 43 & 41 & 47 & \\
\hline III & 22 & 22 & 23 & \\
\hline IV & 4 & 3 & 6 & \\
\hline Creatinine, mg/dL & $1.0 \pm 0.2$ & $1.0 \pm 0.2$ & $1.0 \pm 0.2$ & 0.66 \\
\hline \multicolumn{5}{|l|}{ Medical therapy, \% } \\
\hline ACE inhibitors/ARBs & 43 & 44 & 41 & 0.86 \\
\hline$\beta$-blockers & 33 & 34 & 29 & 0.73 \\
\hline Diuretics & 24 & 25 & 24 & 0.90 \\
\hline \multicolumn{5}{|l|}{ Surgical variables } \\
\hline Bypass time, min & $72 \pm 29$ & $71 \pm 30$ & $74 \pm 28$ & 0.72 \\
\hline Flail leaflet, \% & 61 & 59 & 65 & 0.71 \\
\hline \multicolumn{5}{|c|}{ Posterior leaflet prolapsing segments } \\
\hline Single scallop, \% & 57 & 44 & 82 & 0.007 \\
\hline Multiple scallops, \% & 43 & 56 & 18 & \\
\hline Valve repair, \% & 98 & 97 & 100 & 0.35 \\
\hline
\end{tabular}

flattening in systole. ${ }^{17}$ Despite this standard MMVD presentation and similar MR severity in both groups with similar LV and LA remodelling, there were multiple differences between patients with and without CLI by 3DTOC (table 3, right part). With CLI, the annulus was smaller for all systolic dimensions, anteroposterior $(42.2 \pm 7.1$ vs $47.0 \pm 7.5 \mathrm{~mm}, \mathrm{p}=0.04)$, intercommissural $(36.5 \pm 4.5$ vs $41.4 \pm 5.9 \mathrm{~mm}, \mathrm{p}=0.005)$, circumference $(133 \pm 16$ vs $148 \pm 19 \mathrm{~mm}, \mathrm{p}=0.009)$ and area $(1289 \pm 326$ vs 1619 $\left.\pm 427 \mathrm{~mm}^{2}, \mathrm{p}=0.008\right)$. There was only a trend for smaller annular height with CLI so that the ratio of annular height to intercommissural diameter, a measure of annular saddle shape, was not different with and without CLI. For valvular measurements, there were also marked 3DTOC differences between patients with and without CLI. There was a trend towards smaller volume of prolapse with CLI versus without CLI (1.9 \pm 1.2 vs $4.0 \pm 4.3 \mathrm{~mL}, \mathrm{p}=0.06$ ), which in view of similar maximum height of systolic prolapse suggests smaller prolapse base with CLI. Importantly, valve tissue was markedly smaller with versus without CLI overall (leaflets' area $1574 \pm 409$ vs $2019 \pm 652 \mathrm{~mm}^{2}, \mathrm{p}=0.01$ ), affecting both anterior and posterior leaflet (table 3). These differences are not linked to MR severity as $3 \mathrm{D}$ annular and valvular differences between patients with and without CLI were unchanged stratified by RVol (figure 4). Thus, CLI, as part of the phenotypical spectrum of MMVD, affect patients with single scallop prolapse, limited annular enlargement and relative tissue paucity, irrespective of MR severity.

Table 2 2D echocardiographic baseline characteristics

\begin{tabular}{llll}
\hline & All patients, $\mathbf{n = 4 9}$ & CLI absent, $\mathbf{n = 3 2}$ & CLI present, $\mathrm{n=17}$ \\
\hline LVEF, \% & $63 \pm 7$ & $65 \pm 5$ & $60 \pm 11$ \\
LVEDD, mm & $57 \pm 7$ & $57 \pm 6$ & $57 \pm 6$ \\
LVESD, mm & $36 \pm 5$ & $36 \pm 4$ & $37 \pm 7$ \\
LAVI, mL/m & $60 \pm 17$ & $62 \pm 16$ & $59 \pm 20$ \\
ERO, cm & $0.50 \pm 0.23$ & $0.49 \pm 0.21$ & 0.91 \\
RVol, mL/beat & $85 \pm 48$ & $89 \pm 56$ & $0.54 \pm 0.24$ \\
SPAP, mm Hg & $35 \pm 12$ & $35 \pm 9$ & $79 \pm 29$ \\
Single-leaflet MMVD & $63 \%(31)$ & $59 \%(19)$ & $35 \pm 15$ \\
Bileaflet MMVD & $37 \%(18)$ & $41 \%(13)$ & $71 \%(12)$ \\
\hline
\end{tabular}

CLI, cleft-like indentation; EDD, end-diastolic diameter; ERO, effective regurgitant orifice area; ESD, end-systolic diameter; LAVI, left atrial volume index; MMVD, myxomatous mitral valve disease; RVol, regurgitant volume; SPAP, systolic pulmonary artery pressure. 
Table 3 3D echocardiography annular and valvular measures

\begin{tabular}{|c|c|c|c|c|}
\hline & $\begin{array}{l}\text { All patients, } \\
\mathrm{n}=49\end{array}$ & $\begin{array}{l}\text { CLI absent, } \\
\mathrm{n}=32\end{array}$ & $\begin{array}{l}\text { CLI present, } \\
n=17\end{array}$ & $\mathrm{p}$ Value \\
\hline \multicolumn{5}{|l|}{ Annular dimensions } \\
\hline Intercommissural diameter, mm & $45.4 \pm 7.4$ & $47.0 \pm 7.5$ & $42.2 \pm 7.1$ & 0.04 \\
\hline Anteroposterior diameter, mm & $39.7 \pm 5.5$ & $41.4 \pm 5.9$ & $36.5 \pm 4.5$ & 0.005 \\
\hline Annular height, mm & $6.5 \pm 1.5$ & $6.8 \pm 1.5$ & $6.0 \pm 1.5$ & 0.07 \\
\hline Annular circumference, mm & $143 \pm 18$ & $148 \pm 19$ & $133 \pm 16$ & 0.009 \\
\hline Annular area, $\mathrm{mm}^{2}$ & $1503 \pm 396$ & $1619 \pm 427$ & $1289 \pm 326$ & 0.008 \\
\hline Annular saddle shape, \% & $14.7 \pm 4.1$ & $14.9 \pm 4.1$ & $14.5 \pm 2.4$ & 0.76 \\
\hline \multicolumn{5}{|l|}{ Valvular dimensions } \\
\hline Area of anterior leaflet, $\mathrm{mm}^{2}$ & $909 \pm 285$ & $972 \pm 322$ & $791 \pm 196$ & 0.04 \\
\hline Area of posterior leaflet, $\mathrm{mm}^{2}$ & $956 \pm 364$ & $1048 \pm 409$ & $783 \pm 254$ & 0.02 \\
\hline Valvular area total, $\mathrm{mm}^{2}$ & $1865 \pm 581$ & $2019 \pm 652$ & $1574 \pm 409$ & 0.01 \\
\hline Volume of prolapse, mL & $3.2 \pm 3.6$ & $4.0 \pm 4.3$ & $1.9 \pm 1.2$ & 0.06 \\
\hline Height of prolapse, mm & $7.6 \pm 3.6$ & $8.0 \pm 4.1$ & $7.0 \pm 2.4$ & 0.35 \\
\hline Volume/height prolapse, $\mathrm{cm}^{2}$ & $3.6 \pm 2.5$ & $4.1 \pm 2.9$ & $2.6 \pm 1.1$ & 0.056 \\
\hline
\end{tabular}

Intraobserver and interobserver variability for 3DTOC measurements were small (AP diameter 3.3\% $1.1 \%$ and 5.1\% $\pm 3.7 \%$, annular height $4.6 \% \pm 0.3 \%$ and $1.4 \% \pm 0.6 \%$, annular circumference $2.2 \% \pm 2.8 \%$ and $2.3 \% \pm 6.4 \%$, valvular area $3.3 \% \pm 0.3 \%$ and $7.4 \% \pm 0.8 \%)$ and were similar to those previously reported. ${ }^{17}$

\section{DISCUSSION}

The present study reports on diagnosis and morphological associations of CLI of mitral valve in patients with MMVD undergoing mitral surgery for severe MR. Our study shows that (1) diagnosis of posterior leaflet CLI, which was not feasible by standard 2D echocardiography, was reliable by 3DTOC

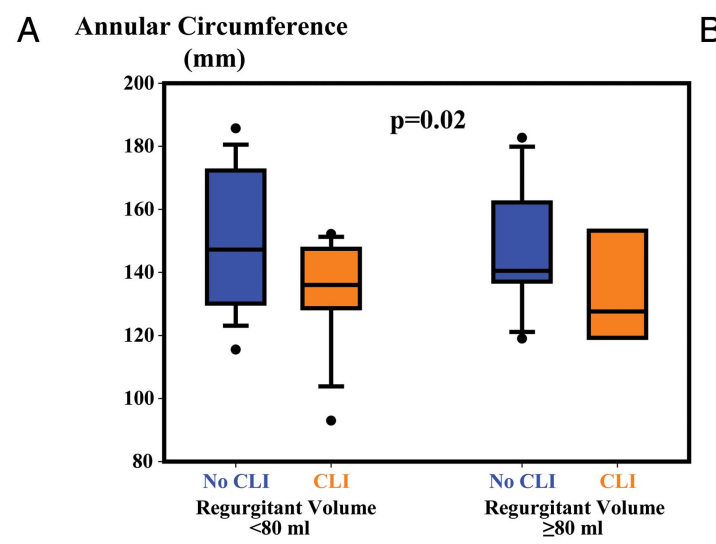

B Annular Area
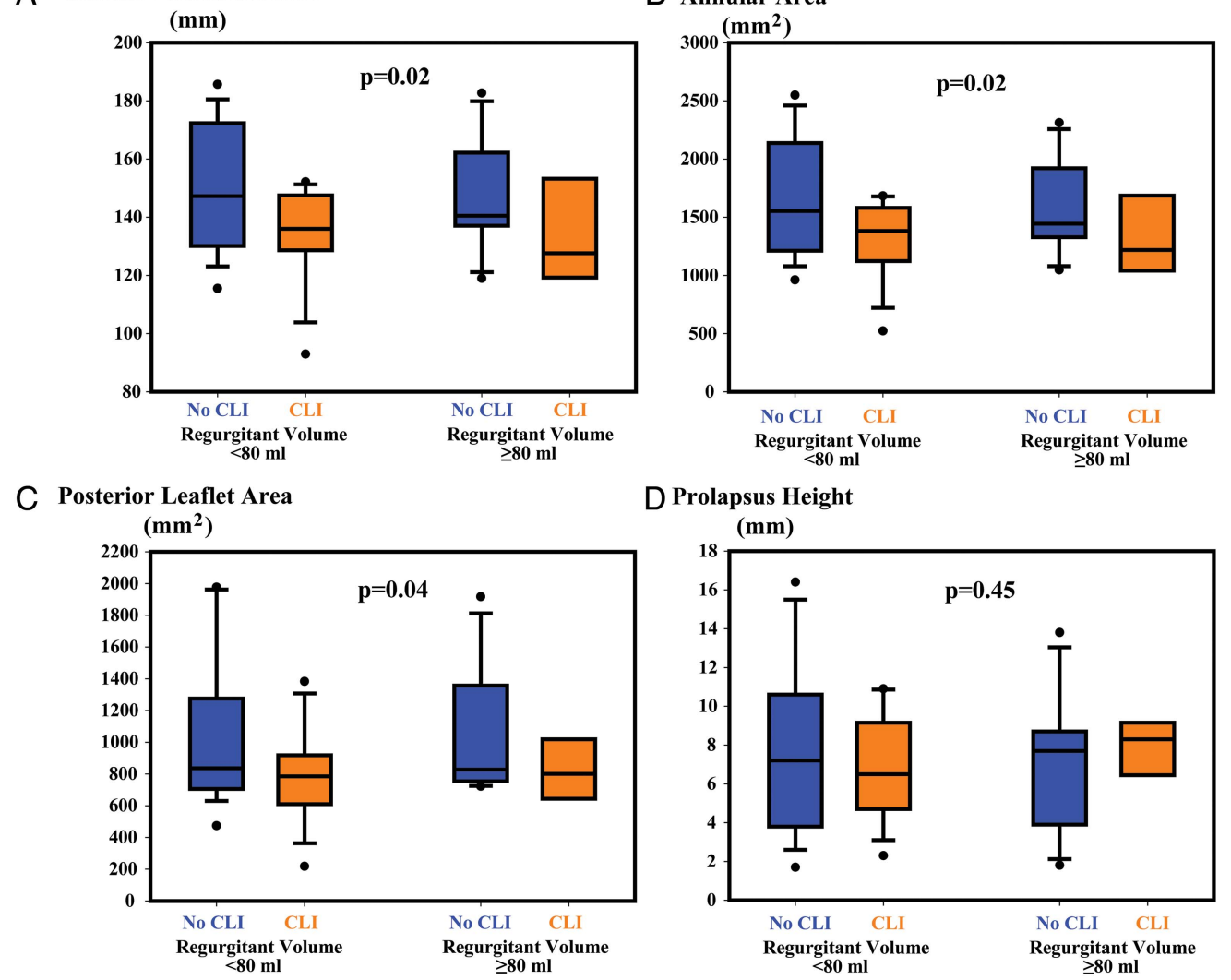

Figure 4 Selected mitral measurements by 3D transoesophageal echocardiography comparing patients with and without CLI and stratified by RVol. The mitral variables represented by box-and-whiskers plots (boxes show $25 \%-75 \%$ quartiles and the whiskers the $95 \% \mathrm{Cl}$ ) are compared between patients without (blue box) and with (orange box) CLI for annular circumference (A), annular area (B), posterior leaflet area (C) and prolapse height (D). The data are stratified by measured RVol (RVol $\geq 80 \mathrm{~mL} /$ beat, left part of the graph; RVol $<80 \mathrm{~mL} / \mathrm{beat}$, right part of the graph). The $p$ value (stratified t test) applies to the global comparison of CLI versus no CLI stratified by RVol. Note that for both strata and all variables represented, except for prolapse height, CLI was associated with smaller annular dimensions and relative tissue paucity compared with patients without CLI. CLI, cleft-like indentations; RVol, regurgitant volume. 
with high sensitivity and specificity; (2) CLI are not an uncommon finding (one-third of patients) in MMVD and requires specific treatment during valve repair and (3) mechanistically, CLI are not explained by excessive annular enlargement that would cause mitral valve stretching or by excessive leaflet prolapse that would cause excess scallop separation. Hence, CLI are part of a global morphological spectrum within MMVD, occurring in the context of single scallop prolapse with insufficient leaflet tissue to cover the marked indentation separating prolapsing and nonprolapsing segments. This wide separation may be accentuated by leaflet resection during valve repair, which underscores the importance of diagnosing CLI by 3DTOC prebypass to conduct proper heart-team communication and valve repair.

\section{Importance of CLI in MMVD}

In the general adult population, CLI of posterior mitral leaflet are rare, with very low prevalence $(\sim 0.1 \%)$ in one echocardiographic study, ${ }^{18}$ but may be slightly higher in normal controls. ${ }^{12}$ CLI have been mentioned in Marfan syndrome ${ }^{19}$ with associated anomalies of the mitral apparatus, such as malrotation of papillary muscles. ${ }^{20}$ In patients with MR, CLI were noted to occur more frequently in MMVD than in patients with other causes of MR. ${ }^{12}$ The quantitative criteria for defining CLI versus normal indentations between posterior mitral scallops remain tentative, but we are confident that using the previously suggested 50\% minimum of adjacent scallops' depth as diagnostic criterion for CLI ${ }^{12} 16$ is concordant with surgical definition of CLI. However, because CLI are part of MMVD morphological spectrum, as shown by our study, CLI prevalence among MMVD populations may depend on the subset enrolled (single vs multi-scallop MMVD in particular) possibly explaining prevalence variability. Careful detailed examination of the entire posterior leaflet by $3 \mathrm{DTOC}$ is the key to diagnosis, but future collaborative studies are warranted to develop quantitative 3D criteria for CLI diagnosis now that appropriate imaging and software allow in-depth analysis of mitral complex. ${ }^{17} 21$

Study of valve characteristics such as CLI has been affected by confusion attached to the label 'cleft'. The typical cleft is a gap at the middle of anterior leaflet, but it has been long known that rarely posterior leaflet may be affected by such true cleft involving central tissue gap. ${ }^{9}$ Diagnosis of this typical pattern of congenital cleft mitral valve was recently enhanced by 3DTOC. ${ }^{10} 1122$ However, use of the term 'cleft' in describing indentations between posterior leaflet scallops, normal or exaggerated, ${ }^{14}$ has been confusing. Hence, we believe, it is essential to separate nosologically CLI from true clefts affecting the posterior leaflet. $^{9} 11{ }^{12}$ In terms of diagnosis, 2DTEE may have difficulty ascertaining typical cleft despite multiplane imaging ${ }^{23} 24$ with even more difficulty with CLI, so that CLI identification is essential in demonstrating 3DTOC usefulness. ${ }^{8}$ Multiple jets and gaping separations between scallops may be clues to CLI, but definite diagnosis requires 3DTOC. Hence, preoperative identification of CLI may be the first strong indication that 3DTOC is important and irreplaceable by 2DTEE. CLI identification takes all its importance in view of recent guidelines that place highquality repair as key to the surgical indication in degenerative MR. ${ }^{5}$ 3DTOC, by identifying in systole CLI of posterior leaflet while the heart is loaded, has definitive advantages over flaccid heart examination and allows quantitative assessment of valvular tissue morphology to define its mechanism. However, 3D pitfalls in interpreting leaflet gaps, such as echo dropouts (annular calcification, improper gain) or oblique imaging planes creating the impression of a fissure not actually present, emphasise proper en-face image acquisition and interpretation for 3DTOC.

\section{Mechanisms of CLI by 3DTOC}

Our working hypothesis that marked annular enlargement may lead to excessive separation of scallops was not verified. The rationale for this hypothesis was previous identification of asymmetric mitral annular enlargement in MMVD, sparing the intertrigonal component (anterior) and dilating the remaining annulus essentially posterior. ${ }^{25}$ However, no measure of annular dimension was larger in patients with CLI, definitely ruling out annular enlargement as potential mechanism. A secondary hypothesis of excess prolapse causing CLI is also not verified as depth and volume of prolapse were not higher in patients with CLI. ${ }^{12}$ Conversely, our study suggests that CLI occur in a specific morphological context, involving single scallop prolapse of posterior leaflet and involving tissue paucity in contrast to patients without CLI. The tissue amount difference between CLI and non-CLI was not limited to posterior leaflet so that overall CLI were not detectable with marked excess global tissue. The prominent indentation may be conformational with native valve tissue paucity or acquired in patients whose response does not involve marked tissue redundancy despite MMVD and severe MR. Indeed, some valve segments may appear normal with isolated severe prolapse or flail segment, usually involving the middle scallop of posterior leaflet. ${ }^{26}$ Acquired accentuation of indentations is also possible, whereby normal positioning of two of the three scallops of posterior leaflet may strain their confluence with the single prolapsing scallop. Normal indentations act much the way pleats do on clothing, allowing leaflets to accommodate the curved shape of valve closure line. ${ }^{27}$ In contrast to patients with CLI, those with multiple prolapsing scallops incur less stretching of normal indentations. Hence, 3DTOC provides mechanistic insights that CLI are associated mostly with single scallop prolapse and insufficient tissue to cover the scallop separation, probably combining conformational and acquired valvular patterns. This data further emphasises the complex combination of various morphological elements in $\mathrm{MMVD}^{21}$ and warrants detailed physiological future studies of annular and valvular dynamics with quantitative MR dynamic assessment to characterise the complete phenotypical manifestations of MMVD.

\section{Practical implication}

Accurate characterisation of MMVD lesion's complexity is critically important to predict feasibility of mitral valve repair and prognosis. $^{28}$ This is increasingly relevant given the growing referral of asymptomatic patients for mitral valve surgery ${ }^{2}$ in whom predictable repair avoiding recurrent $\mathrm{MR}$ is crucial. ${ }^{5} \mathrm{MR}$ of MMVD may be caused by lesions' combination, to which CLI may contribute through extension of the valvular gap, and its satisfactory correction requires addressing all lesions at surgery. ${ }^{29}$ This is particularly important because valvular resection of posterior leaflet middle scallop may accentuate indentation width and cause residual MR and implies careful closure by the surgeon. ${ }^{30}$ Therefore, identification of CLI should be diligent, ${ }^{8}$ and may also be important with the development of catheter-based MR-correction techniques. ${ }^{31}$ In that regard, future resolution improvements of colour-flow imaging may contribute to CLI diagnosis and to the interpretation of its role as a cause of MR.

\section{Limitations of the study}

Population size may have limited our ability to detect borderline differences such as prolapse volume. However, major differences between patients with and without CLI are clearly established 
by our mechanistic 3DTOC study. Larger samples will be necessary in verifying CLI diagnostic accuracy of 3DTOC in clinical practice and the implications of CLI suturing in preventing recurrent MR postrepair. In this study, we could not determine with certainty whether MR occurred through the CLI. With the continued progress of colour-flow imaging in 3DTOC, future studies should focus on defining CLI contribution to lateral or multiple MR jets.

\section{CONCLUSION}

CLI of posterior leaflet are frequent in MMVD, require closure during mitral repair and are identified by 3DTOC with high accuracy. CLI are mechanistically unrelated to excess annular enlargement or excess prolapse, but conversely occur when single scallop prolapse/flail is associated with relative tissue paucity. These 3DTOC data enhance diagnostic and mechanistic comprehension of the diversity of MMVD phenotypical presentation.

\section{Key messages}

\section{What is already known on this subject?}

Recently described cleft-like indentations (CLI) of posterior mitral leaflet in myxomatous mitral valve disease (MMVD) can be associated with mitral regurgitation (MR) and failure of mitral repair.

\section{What might this study add?}

CLI diagnosis is not feasible by standard 2D echocardiography, but is reliable by $3 \mathrm{D}$ transoesophageal echocardiography (3DTOC) and requires specific treatment during valve repair. Mechanistically, CLI are not explained by excessive annular enlargement or excessive leaflet prolapse, but rather by single scallop prolapse and tissue paucity causing excess posterior scallops separation.

\section{How might this impact on clinical practice?} Accurate characterisation of MMVD lesion's complexityessential to define mitral repair feasibility and successunderscores the importance of 3DTOC and potentially its timing. 3DTOC diagnosis of CLI warrants consideration of CLI closure. The finding that tissue paucity is linked to CLI may influence the choice and magnitude of tissue resection during mitral valve repair.

Contributors All authors have contributed to the concept of the study, data collection, data analysis and interpretation, drafting or revision of the manuscript.

Competing interests None declared.

Ethics approval Mayo Clinic Institutional Review Board.

Provenance and peer review Not commissioned; externally peer reviewed.

\section{REFERENCES}

1 lung $B$, Baron $G$, Butchart EG, et al. A prospective survey of patients with valvular heart disease in Europe: The Euro Heart Survey on Valvular Heart Disease. Eur Heart J 2003;24:1231-43.

2 Suri RM, Vanoverschelde JL, Grigioni F, et al. Association between early surgical intervention vs watchful waiting and outcomes for mitral regurgitation due to flail mitral valve leaflets. JAMA 2013;310:609-16.

3 Mohty D, Orszulak TA, Schaff HV, et al. Very long-term survival and durability of mitral valve repair for mitral valve prolapse. Circulation 2001;104:11-7.

4 Suri RM, Schaff HV, Dearani JA, et al. Survival advantage and improved durability of mitral repair for leaflet prolapse subsets in the current era. Ann Thorac Surg 2006;82:819-26
5 Nishimura RA, Otto CM, Bonow RO, et al. 2014 AHA/ACC Guideline for the Management of Patients with Valvular Heart Disease: executive summary: a report of the American College of Cardiology/American Heart Association Task Force on Practice Guidelines. J Am Coll Cardiol 2014;63:2438-88.

6 Enriquez-Sarano M, Freeman WK, Tribouilloy CM, et al. Functional anatomy of mitral regurgitation: accuracy and outcome implications of transesophageal echocardiography. J Am Coll Cardiol 1999;34:1129-36.

7 Lang RM, Tsang W, Weinert $L$, et al. Valvular heart disease. The value of 3-dimensional echocardiography. J Am Coll Cardiol 2011;58:1933-44.

8 Tsang W, Lang RM. Three-dimensional echocardiography is essential for intraoperative assessment of mitral regurgitation. Circulation 2013;128: 643-52.

9 Creech $0 \mathrm{Jr}$, Ledbetter MK, Reemtsma K. Congenital mitral insufficiency with cleft posterior leaflet. Circulation 1962;25:390-4.

10 Seguela PE, Brosset P, Acar P. Isolated cleft of the posterior mitral valve leaflet assessed by real-time 3d echocardiography. Arch Cardiovasc Dis 2011;104: 365-6.

11 Jouni $H$, Driver SL, Enriquez-Sarano M, et al. Cleft posterior mitral leaflet resembling a tri-leaflet mitral valve: a novel phenotypic association with hypertrophic cardiomyopathy. Eur Heart J 2014;35:1623.

12 Ring L, Rana BS, Ho SY, et al. The prevalence and impact of deep clefts in the mitral leaflets in mitral valve prolapse. Eur Heart J Cardiovasc Imaging 2013;14:595-602.

13 McCarthy KP, Ring L, Rana BS. Anatomy of the mitral valve: understanding the mitral valve complex in mitral regurgitation. Eur J Echocardiogr 2010; 11:i3-9.

14 Agricola E, Oppizzi M, Maisano F, et al. Detection of mechanisms of immediate failure by transesophageal echocardiography in quadrangular resection mitral valve repair technique for severe mitral regurgitation. Am J Cardiol 2003;91: 175-9.

15 Zoghbi WA, Enriquez-Sarano M, Foster E, et al. Recommendations for evaluation of the severity of native valvular regurgitation with two-dimensional and Doppler echocardiography. J Am Soc Echocardiogr 2003;16:777-802.

16 La Canna G, Arendar I, Maisano F, et al. Real-time three-dimensional transesophageal echocardiography for assessment of mitral valve functional anatomy in patients with prolapse-related regurgitation. Am J Cardiol 2011;107:1365-74.

17 Grewal J, Suri R, Mankad S, et al. Mitral annular dynamics in myxomatous valve disease: new insights with real-time 3-dimensional echocardiography. Circulation 2010;121:1423-31.

18 Wyss CA, Enseleit F, van der Loo B, et al. Isolated cleft in the posterior mitral valve leaflet: a congenital form of mitral regurgitation. Clin Cardiol 2009;32:553-60.

19 Izgi C, Feray H, Saltan Y, et al. Isolated cleft of the posterior mitral valve leaflet in a patient with Marfan syndrome. Int J Cardiol 2010;145:e102-4.

20 Kent SM, Markwood TT, Vernalis MN, et al. Cleft posterior mitral valve leaflet associated with counterclockwise papillary muscle malrotation. J Am Soc Echocardiogr 2001;14:303-4.

21 Chandra S, Salgo IS, Sugeng $L$, et al. Characterization of degenerative mitral valve disease using morphologic analysis of real-time three-dimensional echocardiographic images: objective insight into complexity and planning of mitral valve repair. Circ Cardiovasc Imaging 2011;4:24-32.

22 Kuperstein R, Feinberg MS, Carasso $S$, et al. The added value of real-time 3-dimensional echocardiography in the diagnosis of isolated cleft mitral valve in adults. J Am Soc Echocardiogr 2006;19:811-14.

23 Lambert AS, Miller JP, Merrick SH, et al. Improved evaluation of the location and mechanism of mitral valve regurgitation with a systematic transesophageal echocardiography examination. Anesth Analg 1999:88:1205-12.

24 Ericksen ML, DiNardo JA. An uncommon source of mitral valve regurgitation. J Cardiothorac Vasc Anesth 2005:19:123-5.

25 Suri RM, Grewal J, Mankad S, et al. Is the anterior intertrigonal distance increased in patients with mitral regurgitation due to leaflet prolapse? Ann Thorac Surg 2009;88:1202-8

26 Anyanwu AC, Adams DH. Etiologic classification of degenerative mitral valve disease: Barlow's disease and fibroelastic deficiency. Semin Thorac Cardiovasc Surg 2007; 19:90-6.

27 Silbiger JJ, Bazaz R. Contemporary insights into the functional anatomy of the mitral valve. Am Heart J 2009;158:887-95.

28 Adams DH, Rosenhek R, Falk V. Degenerative mitral valve regurgitation: best practice revolution. Eur Heart J 2010;31:1958-66.

29 Muraru D, Cattarina M, Boccalini F, et al. Mitral valve anatomy and function: new insights from three-dimensional echocardiography. J Cardiovasc Med 2013:14:91-9.

30 McCarthy PM. Three-dimensional echocardiography is not essential for intraoperative assessment of mitral regurgitation. Circulation 2013;128:653-8.

31 Van Mieghem NM, Piazza N, Anderson RH, et al. Anatomy of the mitral valvular complex and its implications for transcatheter interventions for mitral regurgitation. J Am Coll Cardiol 2010;56:617-26. 